\title{
Avaliação da usabilidade de aplicativos móveis para gestantes com base no System Usability Scale (SUS)
}

\author{
Evaluation of the usability of the mobile application for pregnant women based on the System
} Usability Scale questionnaire (SUS)

Evaluación de la usabilidad de las aplicaciones móviles para embarazadas basada en el cuestionario System Usability Scale (SUS)

\section{Resumo}

Objetivo: Considerando que para um melhor uso e aproveitamento de aplicativos móveis de saúde, é necessário que suas interfaces consigam oferecer funcionalidades que permitam aos usuários realizarem suas tarefas com o mínimo possível de dificuldades em sua utilização, o presente artigo tem como objetivos descrever os aplicativos de saúde móvel sobre gravidez disponíveis para download em smartphones e avaliar a usabilidade com base no instrumento SUS. Método: Trata-se de um estudo transversal e descritivo que utilizou uma busca sistemática de aplicativos nas lojas virtuais Play Store e App Store entre os meses de agosto e setembro de 2020 e, atualizado em março de 2021. As buscas pelos aplicativos foram realizadas por 3 pesquisadoras independentes, de forma simultânea com padronização dos procedimentos. Para a coleta de dados, utilizou-se instrumento desenvolvido especificamente para este estudo. Quanto a avaliação da usabilidade, utilizou-se o instrumento SUS. Resultados: Foram identificados 1.360 aplicativos na loja virtual da Play Store e 1.103 na App Store e, após aplicação dos critérios de seleção, a amostra final foi composta por 56 aplicativos, que foram caracterizados e em seguida foi feita a distribuição de frequência quanto a classificação do questionário de usabilidade SUS. Considerações finais: Os aplicativos oferecem diversos conteúdos, de forma fragmentada e poucos apresentam a fonte das informações disponibilizadas. Quanto a avaliação da usabilidade, a maioria dos aplicativos apresentou uma performance adequada para o uso ao atender as exigências de usabilidade, uma vez que permite que o usuário realize as tarefas com o mínimo de dificuldades.

Palavras-chave: Aplicativos móveis; Saúde móvel; Smartphone; Gravidez; Tecnologia da informação.

\section{Abstract}

Objective: Whereas for better use and enjoyment of mobile health applications, it is necessary that their interfaces are able to offer functionalities that allow users to perform their tasks with the least possible difficulties in their use, this article aims to describe the health applications pregnancy information available for download on smartphones and assess usability based on the SUS instrument. Methodology: This is a cross-sectional descriptive study that used a 
systematic search for apps in the Play Store and App Store virtual stores between the months of August and September 2020 and updated in March 2021. The searches for the apps were performed by 3 independent researchers, simultaneously with standardization of procedures. For data collection, an instrument developed specifically for this study was used. As for the usability assessment, the SUS instrument was used. Results: A total of 1,360 apps was identified in the Play Store virtual store and 1,103 in the App Store. After applying the selection criteria, the final sample consisted of 56 apps, which were characterized, and then the frequency distribution was performed according to the classification. of the SUS usability questionnaire. Final Considerations: The applications offer different content, in a fragmented way and few present the source of the information made available. As for the usability evaluation, most of the applications presented an adequate performance for use in meeting the usability requirements, as it allows the user to perform tasks with minimal difficulties.

Keywords: Mobile Applications; Mobile health; Smartphone; Pregnancy; Information technology.

\begin{abstract}
Resumen
Objetivo: Considerando que para un mejor uso y disfrute de las aplicaciones móviles de salud, es necesario que sus interfaces ofrezcan funcionalidades que permitan a los usuarios realizar sus tareas con las menores dificultades posibles en su manejo, este artículo tiene como objetivos describir las aplicaciones móviles de salud sobre el embarazo disponibles para descargar en smartphones y evaluar la usabilidad con base en el instrumento SUS. Método: Se trata de un estudio transversal y descriptivo que utilizó una búsqueda sistemática de aplicaciones en las tiendas virtuales Play Store y App Store entre los meses de agosto y septiembre de 2020 y, se actualizó el marzo de 2021. Las búsquedas de las aplicaciones fueron realizadas por 3 investigadores independientes, simultáneamente con la estandarización de los procedimientos. Para la recolección de datos se utilizó un instrumento desarrollado específicamente para este estudio. En cuanto a la evaluación de usabilidad, se utilizó el instrumento SUS. Resultados: se han identificados 1.360 aplicaciones en la tienda virtual Play Store y 1.103 en la App Store. Después de la aplicación de los criterios de selección, la muestra final estaba compuesta por 56 aplicaciones, las cuales se caracterizaron y luego se realizó la distribución de frecuencia según la clasificación del cuestionario de usabilidad SUS. Consideraciones finales: Las aplicaciones ofrecen diversos contenidos, de forma fragmentada y pocas presentan la fuente de las informaciones a disposición. En cuanto a la evaluación de usabilidad, la mayoría de las aplicaciones presentaron un desempeño adecuado para su uso al cumplir con los requisitos de usabilidad, puesto que permite al usuario realizar tareas con mínimas dificultades.
\end{abstract}

Palabras clave: Aplicaciones móviles; Salud móvil; Smartphone; Embarazo; Tecnología de la informacíon.

\title{
1. Introdução
}

Com o desenvolvimento das Tecnologias de Informação e Comunicação (TICs), que tem sido rapidamente disseminadas e popularizadas pelo uso de equipamentos móveis como smartphones e tablets com acesso à internet, houve o surgimento de uma gama de aplicativos (apps) que são pequenos programas ou softwares instaláveis em plataformas móveis, desenvolvidos por uma variedade de instituições, indivíduos e organizações, com objetivos que vão desde o entretenimento até a orientação para cuidados e a manutenção da saúde (Lee \& Moon, 2016; Rocha et al., 2017).

Especificamente na área reprodutiva, quando se trata de informações sobre gravidez e cuidados pré-natais, as mulheres grávidas, em especial as primigestas valorizam o uso de fontes on-line para apoiar suas necessidades de informações acerca do período reprodutivo. Apesar das informações também serem adquiridas através de profissionais da saúde, amigos e familiares, a portabilidade e o fácil acesso aos smartphones faz com que as mulheres recorram ao uso desses meios, principalmente através de aplicativos, para obter informações (Halili et al., 2018; Womack et al., 2020).

Os aplicativos que abordam o tema gravidez, parto e cuidados infantis são os mais utilizados nas duas principais lojas virtuais existentes. Segundo estudo que apresentou um conjunto de requisitos para registros pessoais de saúde móveis (mPHRs) para monitoramento de gravidez, extraídos tanto da literatura quanto de aplicativos móveis existentes no mercado, podem ser encontrados mais de 1000 aplicativos com esse tema (Idri et al., 2016). Somente no período gestacional a mulher realiza em média o download de três apps referentes à gravidez, que são usados minimamente meia hora por dia (Frazer et al., 2015).

Gestantes que utilizam apps móveis possuem um melhor nível de adaptação e melhor aceitação da gestação, além de apresentarem mudanças no comportamento, tais como: adoção de postura correta para dormir, se alimentar, realização de sexo 
na gravidez e prática de atividades físicas (Agarwal et al., 2015; Queiroz et al., 2018; Sat \& Sozbir, 2018). Desse modo, desenvolver soluções no formato de aplicativos representa um meio eficaz de atingir o público-alvo.

Contudo, para o melhor uso e aproveitamento desses aplicativos é necessário que suas interfaces consigam oferecer funcionalidades, que permitam aos usuários realizarem suas tarefas com o mínimo possível de dificuldades em sua utilização. Assim, pode-se obter êxito e satisfação em seu uso, aproveitando todas as suas funcionalidades, promovendo uma futura utilização e uma boa experiência pelo usuário (Oliveira, 2016).

Cerca de $80 \%$ dos aplicativos de saúde são abandonados por usuários depois de duas semanas de uso, geralmente pela dificuldade de manuseio e/ou pela deteç̧ão de dados inconsistentes (Telesíntese, 2016). Por isso, a avaliação de um software se apresenta como etapa indispensável para a ampla utilização de aplicativos, já que esse é um processo capaz de aferir o potencial de uma ferramenta tecnológica e a importância dos atributos que ela traz.

A usabilidade faz parte do conjunto de atributos que evidenciam o esforço necessário para utilizar o software, bem como o julgamento individual desse uso por um conjunto de usuários, e é fundamental para garantir a qualidade e aceitação do mesmo (Sperandio, 2017). Visto que, caso seja difícil alcançar um objetivo qualquer, devido à baixa usabilidade da aplicação, o sistema será rapidamente substituído por outro mais usável, assim que sua existência for conhecida pelo público-alvo (Mendes et al., 2006).

Nesse âmbito, surge o System Usability Scale (SUS), instrumento desenvolvido por John Brooke em 1986, que visa medir a usabilidade de diversos produtos e serviços, é de fácil manuseio, capaz de fornecer a visão do usuário sobre o objeto estudado e apresentar resultados confiáveis, independentemente do sistema ou das tarefas (Bangor et al., 2008).

O questionário SUS tem pontos essenciais de indicação de qualidade de software que são imprescindíveis para um bom resultado do produto final, pois a escala de usabilidade abrange um conjunto de aspectos do sistema como a complexidade, necessidade de suporte, interface, entre outros (Brooke, 1996).

A avaliação da usabilidade de aplicativos de saúde móvel sobre gravidez, disponíveis nas lojas virtuais, é de extrema relevância, uma vez que a interface é algo fundamental em um produto, pois ela faz a ligação entre quem usa e o que é disponibilizado pelo sistema em questão (Oliveira, 2016). Além disso, o sucesso e confiabilidade do sistema sofrem influência dessa parte do projeto do software (Sommerville, 2007).

Considerando a fase emergente do uso de aplicativos móveis entre gestantes, acelerado pelo contexto social da pandemia da COVID-19, na qual as pessoas em isolamento social buscam cada vez mais informações online, e o baixo número de investigações sobre a usabilidade de aplicativos móveis voltados para o período gestacional, este estudo surge com o propósito de contribuir com o estado da arte e ampliação do conhecimento sobre a temática.

Assim, o objetivo deste estudo foi: Avaliar a usabilidade de aplicativos de saúde móvel sobre gravidez disponíveis para download em smartphones com base no instrumento SUS.

\section{Metodologia}

Trata-se de um estudo de abordagem quantitativa, de recorte transversal e descritivo. A pesquisa quantitativa é caracterizada pelo emprego da quantificação, tanto nas modalidades de coleta de informações quanto no tratamento delas por meio de técnicas estatísticas (Richardson, 1999). Na pesquisa de corte transversal os dados são coletados em um ponto no tempo, com base em uma amostra selecionada para descrever uma população nesse determinado momento (Marconi \& Lakatos, 2001).

A pesquisa descritiva tem como objetivo principal a descrição das características de determinada população ou fenômeno, ou o estabelecimento de relações entre variáveis (Gil, 2008). Uma de suas características mais significativas aparece na utilização de técnicas padronizadas de coleta de dados. 
Foi realizada uma busca sistemática de aplicativos nas lojas virtuais Play Store e App Store entre os meses de agosto e setembro de 2020 e, atualizado em março de 2021. Os critérios de inclusão dos aplicativos no estudo foram: aplicativos nos idiomas português, inglês e espanhol, gratuitos e que abordassem informações sobre a gravidez em seu título ou descrição. Os critérios de exclusão foram: aplicativos duplicados, de exercícios físicos e alimentação para gestantes, álbum de fotos, editor de fotos, jogos, propagandas, aqueles direcionados somente aos profissionais de saúde, que apresentaram dificuldades para download, acesso restrito e aqueles específicos para registro, como: calculadoras gestacionais para o cálculo da idade gestacional (IG) e da data provável do parto (DPP), registro de movimentos fetais (MF), batimento cardiofetal (BCF) e contador de contrações uterinas.

Para a busca dos aplicativos utilizou-se, em cada loja virtual, as palavras-chave: "gravidez”, "pregnancy”, “el embarazo", "pré-natal e "prenatal”. A Figura 1 apresenta o fluxograma da seleção dos aplicativos móveis.

Figura 1 - Fluxograma da seleção dos aplicativos nas lojas virtuais Play Store e App Store de acordo com os critérios de inclusão. Cuiabá, MT, 2021.

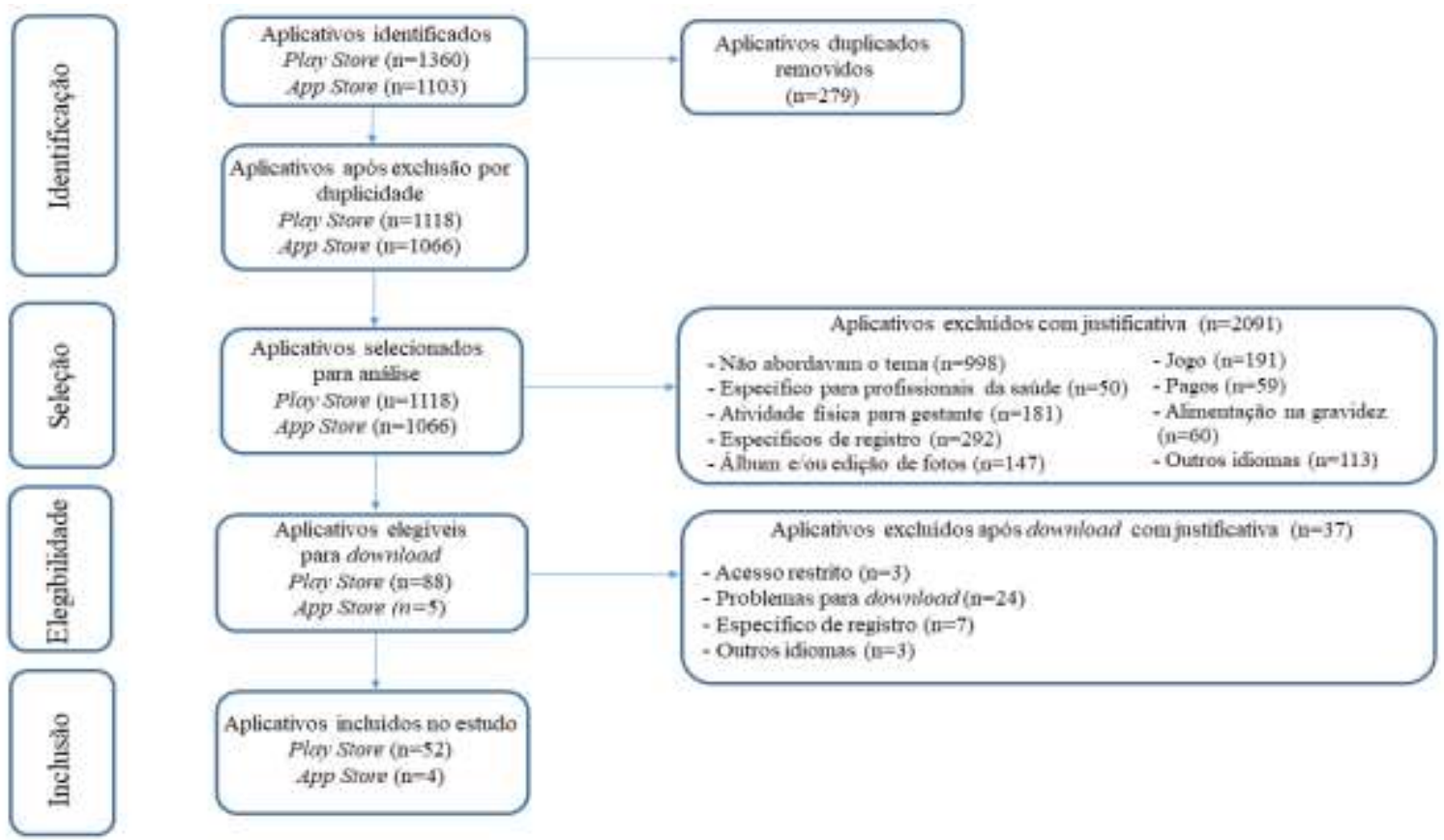

Fonte: Autores (2021).

Cabe destacar que as buscas pelos aplicativos foram realizadas por 3 pesquisadoras independentes, de forma simultânea com padronização dos procedimentos e após a conclusão da busca inicial se reuniram para comparar os achados e eliminar as discrepâncias da amostra visando evitar vieses na triagem dos aplicativos e inferências nos resultados.

Os aplicativos selecionados foram baixados individualmente para os dispositivos (iOS e Android) e manuseados por um tempo médio de 15 minutos. Para a coleta de dados, utilizou-se instrumento desenvolvido especificamente para este estudo, contendo as seguintes variáveis: país de origem, idioma, tipo de linguagem, público-alvo, desenvolvedor, fonte do conteúdo, recursos de mídia e atratividade. Tais variáveis foram utilizadas por se tratarem de informações de fácil acesso disponíveis nos próprios aplicativos e/ou nas lojas virtuais.

Para a avaliação da usabilidade foi utilizado o instrumento System Usability Scale (SUS), por ser um dos mais aceitos devido a sua confiabilidade e validade (Bangor et al., 2008). A escala SUS é composta por 10 questões graduadas em escala tipo Likert, com valores de um a cinco, classificadas respectivamente como "discordo fortemente", "discordo", "não concordo 
nem discordo", "concordo" e "concordo fortemente". O tempo total estimado para responder o questionário variou de 10 a 15 minutos. Para o cálculo do escore, primeiramente é somado o escore de cada item que gera um número único como pontuação total. Cada item contribui em uma escada de 1 a 5 , sendo que para os itens ímpares 1, 3, 5, 7 e 9, o escore individual é a nota recebida menos 1. Para os itens pares 2, 4, 6, 8 e 10, a contribuição é 5 menos a nota recebida. Logo após, multiplica-se a soma de todos os escores por 2,5 para se obter o valor total do instrumento. A partir da pontuação e do cálculo do escore é possível fazer a classificação do SUS em: pior imaginável (até 20,5), pobre (21 a 38,5), mediano (39 a 52,5), bom (53 a 73,5), excelente (74 a 85,5) e melhor imaginável (86 a 100) (Padrini-Andrade et al., 2019).

As variáveis qualitativas foram descritas pelas frequências absoluta (n) e relativa (\%). Para testar a existência de associação entre a variável ordinal (classificação dos escores SUS) e as variáveis nominais (presença de imagens, vídeos, função de registro, diário da gestante, álbum ou editor de fotos e plano de parto) utilizou-se o teste do qui-quadrado de tendência, pois houve interesse em verificar se a prevalência de escores de usabilidade aumenta com a presença de funcionalidades dos apps. Para a realização das análises foram utilizados os softwares MS Excel 2016 e Stata, versão 14.

Não houve necessidade de aprovação no Comitê de Ética em Pesquisa devido ao tipo de pesquisa que utilizou dados analisados serem de domínio público.

\section{Resultados}

Dentre os 56 aplicativos incluídos na amostra, 52 (92,8\%) estavam disponíveis para o sistema operacional Android e 4 (7,2\%) para iOS. No que se refere aos desenvolvedores dos aplicativos identificou-se que 49 (87,5\%) pertenciam a empresas privadas, $4(7,1 \%)$ a particulares, $1(1,8 \%)$ a universidade pública, 1 a universidade privada $(1,8 \%)$ e $1(1,8 \%)$ a organização não governamental sem fins lucrativos.

No que diz respeito ao país de origem, observou-se que o Brasil desenvolveu $15(26,8 \%)$ aplicativos, seguido dos Estados Unidos que desenvolveram 9 (16\%), Espanha desenvolveu 4 (7,1\%) e Rússia 3 (5,4\%). Os seguintes países desenvolveram 1 aplicativo cada: Alemanha, Argentina, Austrália, Bangladesh, Canadá, Colômbia, Eslovênia, Holanda, Índia, Polônia, Suíça, Reino Unido, Ucrânia e Japão. Em 11 aplicativos (19,7\%) não foi possível identificar o país.

$\mathrm{Na}$ Tabela 1 são apresentadas as características dos aplicativos quanto ao idioma, tipo de linguagem, público-alvo, fonte do conteúdo, recursos de mídia e atratividade segundo as lojas virtuais Play Store e App Store. 
Tabela 1 - Caracterização dos aplicativos quanto ao idioma, tipo de linguagem, fonte do conteúdo, recursos de mídia e atratividade segundo as lojas virtuais Play Store e App Store. Cuiabá, MT, Brasil, 2021. (n=56).

\begin{tabular}{|c|c|c|}
\hline \multirow[t]{2}{*}{ Variáveis } & \multirow{2}{*}{$\begin{array}{c}\text { Play Store } \\
\mathrm{n}(\%) \\
\end{array}$} & \multirow{2}{*}{$\begin{array}{c}\text { App Store } \\
\text { n }(\%)\end{array}$} \\
\hline & & \\
\hline \multicolumn{3}{|l|}{ Idioma } \\
\hline Português brasileiro & $24(46,1)$ & $0(0,0)$ \\
\hline Inglês & $19(36,5)$ & $2(50,0)$ \\
\hline Espanhol & $3(5,7)$ & $0(0,0)$ \\
\hline Dois idiomas ou mais & $6(11,5)$ & $2(50,0)$ \\
\hline \multicolumn{3}{|l|}{ Tipo de linguagem } \\
\hline Leiga & $47(90,3)$ & $3(75,0)$ \\
\hline Técnica-científica & $4(7,6)$ & $1(25,0)$ \\
\hline Mista & $1(1,9)$ & $0(0,0)$ \\
\hline \multicolumn{3}{|l|}{ Público-alvo } \\
\hline Gestante & $50(96,2)$ & $4(100,0)$ \\
\hline Gestante e família & $1(1,9)$ & $0(0,0)$ \\
\hline Gestante e profissional de saúde & $1(1,9)$ & $0(0,0)$ \\
\hline \multicolumn{3}{|l|}{ Fonte do conteúdo } \\
\hline Sim & $11(21,1)$ & $1(25,0)$ \\
\hline Não & $41(78,8)$ & $3(75,0)$ \\
\hline \multicolumn{3}{|l|}{ Apresenta imagens } \\
\hline Sim & $46(88,4)$ & $4(100,0)$ \\
\hline Não & $6(11,5)$ & $0(0,0)$ \\
\hline \multicolumn{3}{|l|}{ Apresenta vídeos } \\
\hline Sim & $10(19,2)$ & $4(100,0)$ \\
\hline Não & $42(80,7)$ & $0(0,0)$ \\
\hline \multicolumn{3}{|c|}{$\begin{array}{l}\text { Apresenta função de registro (contração } \\
\text { uterina, BCF, IG, DPP, MF) }\end{array}$} \\
\hline Sim & $19(36,5)$ & $3(75,0)$ \\
\hline Não & $33(63,4)$ & $1(25,0)$ \\
\hline \multicolumn{3}{|l|}{ Apresenta diário da gestante } \\
\hline Sim & $15(28,8)$ & $3(75,0)$ \\
\hline Não & $37(71,1)$ & $1(25,0)$ \\
\hline \multicolumn{3}{|c|}{ Apresenta álbum ou editor de fotos } \\
\hline Sim & $7(13,4)$ & $3(75,0)$ \\
\hline Não & $45(86,5)$ & $1(25,0)$ \\
\hline \multicolumn{3}{|l|}{ Apresenta plano de parto } \\
\hline Sim & $4(7,6)$ & $4(100,0)$ \\
\hline Não & $48(92,3)$ & $0(0,0)$ \\
\hline
\end{tabular}

Fonte: Autores (2021).

Em relação aos conteúdos disponíveis nos aplicativos identificou-se uma diversidade de assuntos acerca do período gestacional, tais como: desenvolvimento fetal, sintomas fisiológicos comuns na gestação, informações sobre as mudanças corporais, prevenção e cuidados com doenças na gravidez, cuidados pré-natais, orientações gerais de cuidados com a pele, intervenções nutricionais, prática de exercícios físicos, consumo de tabaco, álcool e outras substâncias, vacinação e parto.

Quanto aos escores de usabilidade SUS, a Tabela 2 apresenta que 30 (53,5\%) aplicativos foram classificados como "bom" ou "excelente", ao passo que 20 (35,7\%) receberam a classificação como "melhor impossível". 
Tabela 2 - Distribuição de frequência quanto a classificação do questionário de usabilidade SUS. Cuiabá, MT, Brasil, 2021. $(n=56)$.

\begin{tabular}{lcc}
\hline \multicolumn{1}{c}{ Classificação } & n (\%) & \% acumulada \\
\hline Pobre & $2(3.6)$ & 100.0 \\
Mediano & $4(7,1)$ & 60,7 \\
Bom & $12(21,5)$ & 21,4 \\
Excelente & $18(32,1)$ & 53,5 \\
Melhor imaginável & $20(35,7)$ & 96,4 \\
Total & $56(100,0)$ & \\
\hline
\end{tabular}

Fonte: Autores (2021).

Quando analisada pelo teste Qui-quadrado, a associação da classificação do escore SUS com as variáveis nominais dicotômicas: presença de imagens, vídeos, função de registro, diário da gestante, álbum ou editor de fotos e plano de parto não foi estatisticamente significativa, com $\mathrm{p}$-valor $=0,882 ; \mathrm{p}$-valor $=0,641 ; \mathrm{p}$-valor $=0,747 ; \mathrm{p}$-valor $=0,625 ; \mathrm{p}$-valor $=0,362$ e $\mathrm{p}$ valor $=0,498$; respectivamente.

\section{Discussão}

A vasta gama de aplicativos encontrados no desenvolvimento deste estudo está, possivelmente, relacionada à complexidade do período gestacional que é permeado por dúvidas, temores e curiosidades perante as mudanças corporais e psíquicas que o acompanham. E, motivadas pela necessidade de obter conhecimentos, seja pela inexperiência ou pelo desejo de compartilhar suas vivências com outras pessoas, as mulheres buscam em sites, aplicativos (app) e redes sociais informações sobre a gestação atual ou futura (Queiroz et al., 2021).

Para Goetz et al. (2017) o uso de apps mHealth, sem dúvida, abre novos espaços para o progresso futuro. As informações seguras e advindas de fontes confiáveis inspiram credibilidade, o que denota a importância desses aspectos para a escolha da via de informação que causa interesse e que será utilizado pelo público-alvo (Queiroz et al., 2021).

A maioria dos aplicativos não apresentaram a fonte do conteúdo, assim como, em um estudo feito por Goetz et al. (2017), menos de 15\% dos 1.800 apps disponíveis no sistema operacional da Apple foram considerados potencialmente úteis por obstetras-ginecologistas, pois a maioria não possuía fonte de informação.

Outro fator importante citado por Gomes et al. (2019), ao considerar as condições socioculturais da nossa população, é a necessidade de desenvolvimento de aplicativos em português. A predominância de aplicativos com o idioma português brasileiro, demonstra a alta demanda destes dispositivos por gestantes no Brasil.

A maioria dos aplicativos analisados tem como público-alvo as gestantes. Nos últimos anos muito tem se falado sobre a importância do envolvimento consciente e ativo do pai/parceiro no período pré-natal, não só como apoio emocional à gestante, mas também para a criação de vínculo afetivo com o bebê (Henz et al., 2017). O Pré-Natal do Parceiro surgiu em 2016 com o intuito de inserir o pai/parceiro no pré-natal, para compreender as mudanças que ocorrem com a gestante nesta fase, permitindo que o companheiro possa compartilhar desses momentos com a mulher (Brasil, 2016).

Segundo Halili et al. (2018), mulheres grávidas, principalmente as mais novas, valorizam muito o uso de fontes online para apoiar suas necessidades de informações no período gravídico visto que, a comunicação durante o pré-natal se apresenta cada vez mais superficial, escassa e breve.

A assistência pré-natal caracteriza-se por um conjunto de práticas de saúde que agregam ações de promoção da saúde e prevenção de agravos, diagnósticas e curativas, com objetivo de assegurar a saúde e o bem-estar da mulher e do concepto, e o 
bom desfecho da gestação para ambos (OMS, 2016). Contudo, pesquisas nacionais que avaliaram a adequação desta assistência no Brasil, a partir dos requisitos preconizados pelo Ministério da Saúde, apontam que apesar dos avanços, permanece a necessidade de melhorar a qualidade do cuidado ofertado (Domingues et al., 2015; Leal et al., 2020), o que consequentemente pode estar relacionado a busca das mulheres por outros meios de informação para sanar as suas dúvidas, tais como os aplicativos móveis. Estudos apontam que inúmeras práticas rotineiras carecem de orientações específicas na gravidez, como alimentação saudável, cuidados relativos à saúde ocupacional, prática de esportes, exposição a agentes nocivos como medicamentos sintomáticos etc. (Gonçalves, 2016; Oliveira \& Santos, 2018).

Dentre os vários conteúdos abordados em apps para gravidez, os informativos constituem a maior parte deles, os mais comuns são a respeito do desenvolvimento fetal, medida uterina, calculadora para data provável do parto, temporizador de contração e alguns avançados permitem a monitorização fetal (Lee \& Moon, 2016; Tripp et al., 2014).

Poucos aplicativos abordaram o Plano de Parto (PP) que é um instrumento onde as gestantes expressam os cuidados que desejam durante o seu trabalho de parto, parto e pós-parto. $\mathrm{O}$ uso do plano de parto é recomendado pela Organização Mundial da Saúde (OMS) e pelo Ministério da Saúde no Brasil desde 2011, após a implantação do Programa Rede Cegonha.

No entanto, ainda existe certo desconhecimento tanto das gestantes, quanto dos profissionais de saúde, muitas mulheres nunca preencheram um PP, ou sequer ouviram falar sobre o documento, por isso, a importância de incluir esse documento tanto na rotina da assistência ao pré-natal quanto nos aplicativos de saúde voltados a gravidez e cuidados pré-natais (Anderson et al., 2017; Mouta et al., 2017; Silva et al., 2019).

No que tange à avaliação da usabilidade, os resultados apontam para a viabilidade dos aplicativos analisados referente às suas finalidades. Para Brooke (1996), os pontos essenciais de indicação de qualidade de softwares contidas no questionário SUS são imprescindíveis para um bom resultado do produto final, pois a escala de usabilidade abrange um conjunto de aspectos do sistema como a complexidade, necessidade de suporte, interface, entre outros.

O escore SUS mostrou que a maioria dos aplicativos apresentou uma performance adequada para o uso ao atender as exigências de usabilidade, assim como em outros estudos que utilizaram a escala para avaliar aplicativos de saúde e que também obtiveram resultados favoráveis (Cavalcanti et al., 2021).

O teste de usabilidade permite entender fatores subjetivos que determinem o uso efetivo do sistema, e que podem contribuir para o aperfeiçoamento e a experiência da interação humano-computador (IHC), assim como a sugestão de melhorias no aplicativo quanto ao conteúdo, pois as informações podem ser verificadas e atualizadas em novas versões para um melhor aperfeiçoamento da ferramenta (Andrade et al., 2019; Cavalcanti et al., 2021).

A facilidade de acessar, manusear e localizar informações são aspectos imprescindíveis e motivadores para o uso de aplicativos por gestantes, além da diversidade de informações que oferece ao público-alvo (Queiroz et al., 2021). Mulheres que utilizam aplicativos para acompanhamento da gestação, preferem uma interface fácil de usar e sem a interferência de questões técnicas demoradas (Goetz et al., 2017). Os de fácil manuseio e autoexplicativos são os que mais causam motivações para uso e para mudanças atitudinais nos usuários (Queiroz et al., 2021).

Assim, a usabilidade torna-se fundamental para o aumento da qualidade e competitividade de um aplicativo e é necessária para melhorar a qualidade e aceitação do software (Delgado et al., 2019).

\section{Considerações Finais}

A partir dos resultados deste estudo pode-se concluir que a maioria dos aplicativos para gestantes disponíveis para smartphone foram desenvolvidos por empresas privadas, sediadas em países de todo o mundo, principalmente no Brasil e nos Estados Unidos, disponibilizados nos principais idiomas e com conteúdo informativos que se apresentam de forma fragmentada, ou seja, não contemplam informações completas que condizem com um cuidado holístico e de acordo com as 
necessidades reais das gestantes, fazendo com que tenham que baixar mais de um aplicativo para obter mais informações sobre a gestação.

O fato de poucos aplicativos apresentarem a fonte do conteúdo nas informações disponibilizadas faz refletir sobre a necessidade de existir um maior rigor científico por parte dos desenvolvedores, uma vez que a disponibilização de conteúdos de origem confiável, atualizado e baseado em evidências científicas implica na construção de um sujeito empoderado, diligente e transformador dos cuidados com sua saúde.

Quanto a avaliação da usabilidade, o escore SUS mostrou que a maioria dos aplicativos apresentou uma performance adequada para o uso ao atender as exigências de usabilidade, uma vez que permite que o usuário realize as tarefas com o mínimo de dificuldades, contribuindo para utilizações futuras, satisfação e boa experiência de uso pelo público-alvo.

Embora a usabilidade tenha sido avaliada de forma positiva, este estudo permitiu identificar oportunidades de melhorias em diversos aplicativos encontrados no mercado, o que indica uma possibilidade de aperfeiçoamento ou construção de novos aplicativos voltados para as necessidades reais das mulheres no período gravídico-puerperal.

\section{Referências}

Agarwal, K. C., Davis, L. D., Williams, L., Davey, R., Cox, R., \& Clerke, A. (2015). Development and Pilot Testing of the Eating4two Mobile Phone App to Monitor Gestational Weight Gain. JMIR Mhealth Uhealth, 3(2), 44. 10.2196/mhealth.4071.

Anderson, C. M., Monardo, R., Soon, R., Lum, J., Tschann, M., \& Kaneshiro, B. (2017). Patient Communication, Satisfaction, and Trust Before and After Use of a Standardized Birth Plan. Hawai'i J Med Public Health, 76(11), 305-9.

Andrade, L. P., Balda, R. C. X., Areco, K. C. N., Paiva, P. B., Nunes, M. V., Marba, S. T. M., Carvalho, W. B., Rugolo, L. M. S. S., Almeida, J. H. C., Procianoy, R. S., Duarte, J, L. M., Rego, M. A. S., Ferreira, D. M. L. M., Filho, N. A., Guinsburg, R., Diniz, E. M. A., Santos, J. P. F., Testoni, D., Silva, N. M. M., Gonzales, M. R. C., Silva, R. V. C., Meneses, J., Ferri, W. A. G., Silva, R. P., \& Bomfim, O. (2019). Avaliação da usabilidade de um sistema de informação em saúde neonatal segundo a percepção de usuário. Revista Paulista de Pediatria, 37(1), 90-96. http://dx.doi.org/10.1590/19840462/;2019;37;1;00019.

Bangor, A., Kortum, P. T., \& Miller, J. T. (2008). An empirical evaluation of the system usability scale. International J Hum Comput Interact, 24(6), 574-594.

Brasil. (2016). Ministério da Saúde. Guia do Pré-Natal do Parceiro para Profissionais da Saúde. Ministério da Saúde, 2016. http://portalarquivos2.saude.gov.br/images/pdf/2016/agosto/11/guia_PreNatal.pdf Acesso em 20 de outubro de 2018.

Brooke, J. (1996). SUS: a quick and dirty usability scale. Usability evaluation in industry, Taylor \& Francis; $189-94$.

Cavalcanti, H. G. O., Bushatsky, M., Barros, M. B. S. C., Melo, C. M. C. S., \& Filho, A. J. F. D. (2021). Avaliação da Usabilidade de um aplicativo móvel para detecção precoce do câncer pediátrico. Revista Gaúcha de Enfermagem, 42. https://doi.org/10.1590/1983-1447.2021.20190384.

Delgado, G. V., Carvalho, R. B., Castro, J. M., \& Leite, R. S. (2019). Empoderamento por meio de aplicativos de saúde: Um Estudo de Caso de um Aplicativo para Gestantes. Revista de Administração da UNIMEP, 17(1), 89, http://dx.doi.org/10.29397/reciis.v12i1.1365.

Domingues, R. M., Viellas, E. F., Dias, M. A., Torres, J. A., Theme-Filha, M. M., Gama, S. G., \& Leal, M. C. (2015). Adequação da assistência pré-natal segundo as características maternas no Brasil. Revista Panamericana de Salud Pública, 37(3), 142-5.

Frazer, C., Hussey, L., Bosch, E., \& Squire, M. (2015). Pregnancy App: A Closer look at the implications for child bird educators. International Journal of Childbirth Education, 30(3).

Gil, A. C. (2008). Como elaborar projetos de pesquisa (4a ed.) Atlas.

Goetz, M., Muller, M., Matthies, L. M., Hansen, J., Doster, A., Szabo, A., Frohlich, J. P., Abele, H., Sohn, C., Wallwiener, M., \& Wallwiener, S. (2017). Perceptions of Patient Engagement Applications During Pregnancy: A Qualitative Assessment of the Patient's Perspective. JMIR Mhealth Uhealth, 5(5), 73. 10.2196/mhealth.7040.

Gomes, M. L., Rodrigues, I. R., Moura, N. S., Bezerra, K. C., Lopes, B. B., Teixeira, J. J. D., Vasconcelos, C. T. M., \& Oriá, M. O. B. (2019). Avaliação de aplicativos móveis para promoção da saúde de gestantes com pré-eclâmpsia. Acta paulista de enfermagem, 32(3), 275-81. https://doi.org/10.1590/19820194201900038 .

Gonçalves, S. M. (2009). Proposta de Requisitos para Protótipo de Software em m-Health como apoio à Promoção da Saúde Materno-Infantil em Conformidade com as Políticas Públicas de Saúde. Dissertação (Mestrado em Promoção da Saúde) - Unicesumar, Maringá-PR.

Halili, L., Liu, R., Hutchinson, K. A., Semeniuk, K., Redman, L. M., \& Adamo, K. B. (2018). Development and pilot evaluation of a pregnancy-specific mobile health tool: a qualitative investigation of SmartMoms Canada. Medical Informatics and Decision Making, Canada, 8(95). https://doi.org/10.1186/s12911-018-0705-8.

Henz, G. S., Medeiros, C. R. G., \& Salvadora, M. (2017). A Inclusão paterna durante o pré-natal. Revista de Enfermagem e Atenção à Saúde, 6(1), 52-66. https://doi.org/10.18554/reas.v6i1.2053. 
Idri, A., Bachiri, M., \& Alemán, J. L. F. (2016). A framework for evaluating the software product quality of pregnancy monitoring móbile personal health records. Journal of Medical Systems, 40(3), 40-50. 10.1007 / s10916-015-0415-z.

Leal, M. C., Esteves-Pereira, A. P., Viellas, E. F., Domingues, R. M. S. M., \& Gama, S. G. N. Assistência pré-natal na rede pública do Brasil (2020). Revista de Saúde Pública, 54(8).

Lee, Y., \& Moon, M. (2016). Utilization and content evaluation of móbile applications for pregnancy, birth, and child care. Healthcare Informatics Research, 22(2), 73-80. http://dx.doi.org/10.4258/hir.2016.22.2.73.

Marconi, M. A., \& Lakatos, E. M. (2001). Metodologia do trabalho científico (6a ed.) Atlas.

Mendes, E., Mosley, N., \& Counsell, S. (2006). "The Need for Web Engineering: An Introduction”.

Mouta, R. J. O., Silva, T. M. A., Melo, P. T. S., Lopes, N. S., \& Moreira, V. A. (2017). Plano de parto como estratégia de empoderamento feminino. Revista baiana de enfermagem, 31(4), 20275.

Oliveira, G. M.; Santos, L. F. (2018). Uso de aplicativos para dispositivos móveis no processo de educação em saúde: reflexos da contemporaneidade. Revista Observatório, Palmas, 4(6), 826-844. https://doi.org/10.20873/uft.2447-4266.2018v4n6p826.

Oliveira, J. M. (2016). Avaliação de usabilidade em aplicativos para apoio à participação em eventos. Curso de Ciência da Computação (Dissertação), Universidade Federal de Viçosa, Minas Gerais.

Organização Mundial da Saúde (2016). Recommendations on antenatal care for a positive pregnancy experience. Geneva, OMS. https://apps.who.int/iris/bitstream/handle/10665/250796/9789241549912-eng.pdf.

Queiroz, F. F. S. N., Brasil, C. C. P., Silva, R. M. Collares, P. M. C., \& Filho, J. E. V. (2018). Os significados atribuídos pelas gestantes ao aplicativo GestAção. Investigação Qualitativa em Saúde, 2.

Queiroz, F. F. S. N., Brasil, C. C. P., Silva, R. M., Bezerra, I. C., Collares, P. M. C., \& Filho, J. E. V. (2021). Avaliação do aplicativo "Gestação" na perspectiva da semiótica: o olhar das gestantes. Ciência \& Saúde Coletiva, 26(2), 485-492. 10.1590/1413-81232021262.41002020.

Richardson, R. J. (1999). Pesquisa social: métodos e técnicas (3a ed.) atlas.

Rocha, F. S., Santana, E. B., Silva, E. S., Carvalho, J. S. M., \& Carvalho, F. L. Q. (2017). Uso de app para a promoção de cuidados á saúde. Seminário de tecnologias aplicadas em educação e saúde, UNEB.

Sat, S. O., \& Sozbir, S. Y. (2018). Use of mobile applications and blogs by pregnant women in Turkey and the impacto on adaptation to pregnancy. Journals Elsevier Midwifery, 62, 273-277. 10.1016/j.midw.2018.04.001.

Silva, A. B., Assumpção, A. M. B., Filha, I. G. A., Regadas, C. T., Castro, M. C., Silva, C. R. A., Assumpção, M. R., Santos, R. C. B., Silvério, T. O., Santos, P. B., Silva, D. A., Paulino, B. V., \& Pastorelli, P. P. L. (2019). Adaptação transcultural do aplicativo Zero Mothers Die para dispositivos móveis no Brasil: contribuições para a saúde digital com abordagem do cuidado centrado na e-gestante. Revista brasileira de saúde materno infantil, Recife, 19(4), 763-775, http://dx.doi.org/10.1590/1806-93042019000400002.

Sommerville, I. (2007). Engenharia de software. (8a ed.), Addison Wesley, 592p.

Sperandio, M. R. C. (2017). Ensino de ciências por investigação para professores da educação básica: dificuldades e experiências de sucesso em oficinas pedagógicas. Dissertação (Mestrado em Ensino de Ciências Humanas, Sociais e da Natureza) - Universidade Tecnológica Federal do Paraná, Londrina, 237f. Telesíntese. (2016). Telecomunicação, Internet e Tics. https://www.telesintese.com.br/tic-domicilios-2016/.

Tripp, A., Hainey, K., Liu, A., Poulton, A., Peek, M., Kim, J., \& Nanan, R. (2014). An emerging model of maternity care: Smartphone, midwife, doctor? Women and Birth - Elsevier Australia, (27), 64-67. 10.1016/j.wombi.2013.11.001.

Womack, J. J., Anderson, L. N., \& Ledfor, C. J. W. (2020). Presence of Complex and Potentially Conflicting information in prenatal mobile apps. Health promotion practice, 21(2), 238-245. https://doi.org/10.1177/1524839918796216. 